\title{
Lifetime of individual crystals in a rock: quantifying crystal size distributions and concentration gradients using the phase-field method
}

\author{
J. KUNDIN ${ }^{1}$, I.STEINBACH ${ }^{1}$, S. CHAKRABORTY ${ }^{2 *}$
}

${ }^{1}$ ICAMS, Ruhr-Universität Bochum, Germany ${ }^{2}$ Inst. für Geol., Min. \& Geoph., Ruhr-Universität Bochum, Bochum, Germany (Julia.Kundin@rub.de ; Ingo.Steinbach@rub.de, *Sumit.Chakraborty@rub.de. *correspondence)

Phase diagrams are an integral part of petrology and are widely used to determine the pressure and temperature conditions at which minerals crystallized. However, the textural context in which these minerals occur are not accounted for in such calculations. In contrast, microstructural features such as crystal size distribution (CSD) are used to infer the timescale and mechanisms of crystal growth in rocks (e.g. [1,2]). A quantitative connection between the energetics that governs equilibrium phase relations and texture maturation (including lifetime and composition record of particular crystals) that results in the observed CSD is unclear until now. The phase-field approach provides a means to study such connections. It considers the surface energy in the overall free energy balance and treats phase boundaries as moving diffuse interface regions in a thermodynamically consistent manner (e.g. see [3] for a review). As a result, calculations can predict not only the chemistry and modal abundance of stable phases, but also the shape and size of particular crystals that are stable (and the CSD, as an integrated result of these). Here, we will show an example of the simulation of such processes in a two-component, two-phase system to illustrate how the lifetime of a particular crystal of a phase in a system may be quite different from the duration of existence of the phase as a whole.

[1] Marsh, B. (1988) Contrib. Mineral. Petrol. 99: 277-291.

[2] Cashman, K. et al. (1988) Contrib. Mineral. Petrol. 99: 292305.

[3] Steinbach, I. (2009) MSMSE 17, 073001 (31pp). 